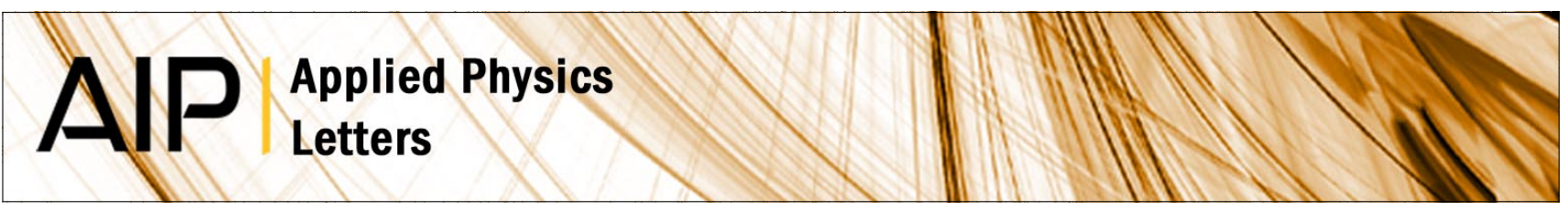

\title{
Magnetic memory based on La0.7Ca0.3MnO3/YBa2Cu3O7/La0.7Ca0.3MnO3 ferromagnet/superconductor hybrid structures
}

N. M. Nemes, C. Visani, C. Leon, M. Garcia-Hernandez, F. Simon et al.

Citation: Appl. Phys. Lett. 97, 032501 (2010); doi: 10.1063/1.3464960

View online: http://dx.doi.org/10.1063/1.3464960

View Table of Contents: http://apl.aip.org/resource/1/APPLAB/v97/i3

Published by the American Institute of Physics.

Additional information on Appl. Phys. Lett.

Journal Homepage: http://apl.aip.org/

Journal Information: http://apl.aip.org/about/about_the_journal

Top downloads: http://apl.aip.org/features/most_downloaded

Information for Authors: http://apl.aip.org/authors

\section{ADVERTISEMENT}

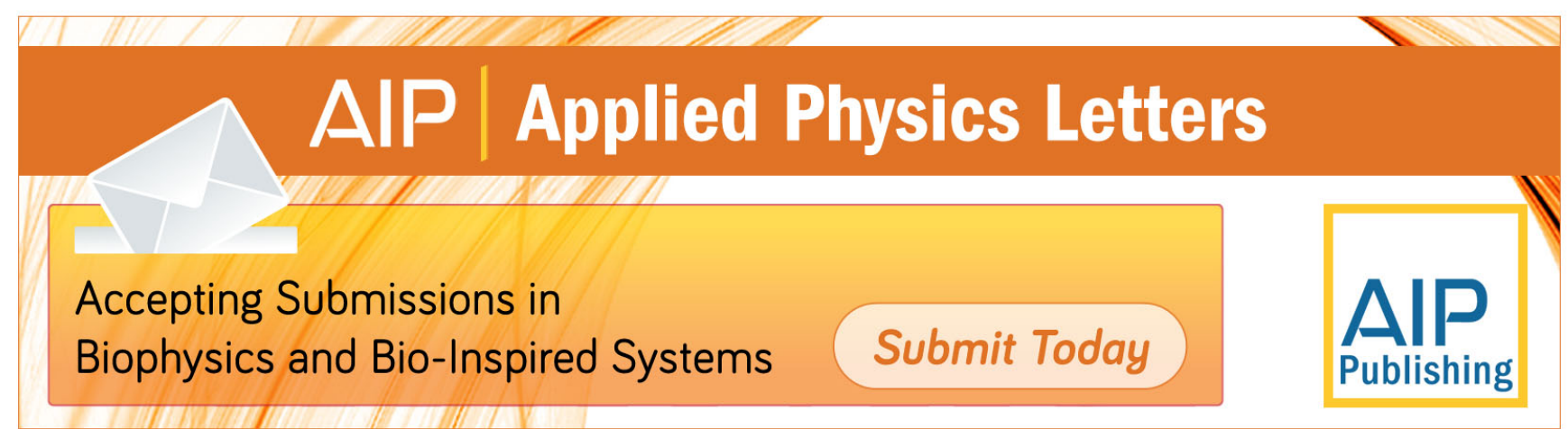




\title{
Magnetic memory based on $\mathrm{La}_{0.7} \mathrm{Ca}_{0.3} \mathrm{MnO}_{3} / \mathrm{YBa}_{2} \mathrm{Cu}_{3} \mathrm{O}_{7} / \mathrm{La}_{0.7} \mathrm{Ca}_{0.3} \mathrm{MnO}_{3}$ ferromagnet/superconductor hybrid structures
}

\author{
N. M. Nemes, ${ }^{1}$ C. Visani, ${ }^{1}$ C. Leon, ${ }_{4}^{1}$ M. Garcia-Hernandez, ${ }^{2}$ F. Simon, ${ }^{3}$ T. Fehér, ${ }^{3}$ \\ S. G. E. te Velthuis, ${ }^{4}$ A. Hoffmann, ${ }^{4}$ and J. Santamaria ${ }^{1, a)}$ \\ ${ }^{1}$ Dpto. Fisica Aplicada III, GFMC, Universidad Complutense de Madrid, Campus Moncloa, \\ 28040 Madrid, Spain \\ ${ }^{2}$ Instituto de Ciencia de Materiales de Madrid, Consejo Superior de Investigaciones Cientificas, \\ 28049 Cantoblanco, Spain \\ ${ }^{3}$ Department of Physics, Budapest University of Technology and Economics and Condensed Matter Physics \\ Research Group, Hungarian Academy of Sciences, Budapest H-1521, Hungary \\ ${ }^{4}$ Materials Science Division, Argonne National Laboratory, Argonne, Illinois 60439, USA
}

(Received 7 May 2010; accepted 26 June 2010; published online 20 July 2010)

\begin{abstract}
We report a memory concept utilizing ferromagnet/superconductor/ferromagnet $\mathrm{La}_{0.7} \mathrm{Ca}_{0.3} \mathrm{MnO}_{3} / \mathrm{YBa}_{2} \mathrm{Cu}_{3} \mathrm{O}_{7} / \mathrm{La}_{0.7} \mathrm{Ca}_{0.3} \mathrm{MnO}_{3}$ thin film hybrid structures. The orientation of the magnetic field with respect to the ferromagnetic easy axis has a strong effect on superconductivity as indicated by a strong variation in the magnetoresistance (MR). MR can be controlled by rotating a small magnetic field applied in the plane of the film in a way that is determined by the in-plane biaxial magnetic anisotropy. The proposed memory device has the advantages of superconducting detection elements (fast response and low dissipation), small (100-150 Oe) writing fields, and resistance read-out without need for applied field. (C) 2010 American Institute of Physics. [doi:10.1063/1.3464960]
\end{abstract}

Superconductor-based electronics is a long pursued concept, because of its advantages, such as power consumption and switching time. Apart from the operation temperature, one of the major obstacles is the difficulty of implementing a dense memory technology. Ferromagnetic/superconductor/ ferromagnetic $(\mathrm{F} / \mathrm{S} / \mathrm{F})$ heterostructures were proposed as possible pathways toward next generation spintronic devices and memories ${ }^{1-3}$ exploiting the large magnetoresistance (MR) of proximity coupled structures or their potential superior spinpolarized transport performance due to longer spin decoherence times. ${ }^{4}$ At F/S interfaces, the superconducting order parameter penetrates short distances into the ferromagnet, over which Cooper pairs directly feel the exchange interaction. This results in a strong interplay between both types of longrange order. In this framework, the possibility of enhanced superconductivity modulation by controlling the relative magnetic alignment (parallel, $\mathrm{P}$ versus antiparallel, AP) of the $\mathrm{F}$ layers in $\mathrm{F} / \mathrm{S} / \mathrm{F}$ double junctions ${ }^{5-13}$ gives rise to interesting concepts for implementing memory devices.

In nonsuperconducting magnetoresistive memory elements currently employed in commercially available devices, the magnetization of one freely rotatable magnetic layer is toggled via a well-defined sequence of current pulses through two perpendicular conducting wires, which results in an in-plane rotation of the effective Oersted field. The substitution of the metallic $\mathrm{N}$ layer by a superconductor provides a possible approach toward a superconducting memory, with the advantage of an anticipated large MR ratio due to the averaging of the exchange field over the coherence volume in the AP configuration. However, the experimental realizations of this effect in samples based on low $T_{c}$ superconductors and transition metal ferromagnets showed very narrow temperature intervals (a few millikelvin) separating the criti-

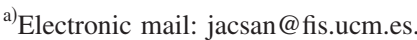

cal temperatures in the $\mathrm{P}$ and AP configurations, and small MR values, drawbacks that yet limit the practical usage of this memory concept.

Here, we show that the interplay between magnetic anisotropy and $\mathrm{F} / \mathrm{S}$ intertalk in oxide based $\mathrm{La}_{0.7} \mathrm{Ca}_{0.3} \mathrm{MnO}_{3}(\mathrm{LCMO}) / \mathrm{YBa}_{2} \mathrm{Cu}_{3} \mathrm{O}_{7}(\mathrm{YBCO}) /$ $\mathrm{La}_{0.7} \mathrm{Ca}_{0.3} \mathrm{MnO}_{3}$ (LCMO) spin switches leads to a different concept of superconducting memory where the high and low resistance states are controlled by rotating a small, in-plane magnetic field. The advantages of the present approach are twofold; first the structure is not proximity coupled, second the anisotropy is biaxial with easy magnetic axes along the [110] substrate directions. This system behaves as an inverse superconducting spin switch with superconductivity favored when the F layers are P. The (magneto)resistance, with much larger values than in ordinary superconducting spin switches, closely follows the AP alignment with a high resistance state in the AP configuration and a low resistance state under $\mathrm{P}$ alignment (as opposed to the ordinary superconducting spin switch). ${ }^{13-15}$

We grew $\mathrm{F} / \mathrm{S} / \mathrm{F}$ trilayers with fixed $15 \mathrm{~nm}$ thick top and bottom LCMO layers on (100) $\mathrm{SrTiO}_{3}$ (STO) substrates by sputter deposition in pure oxygen. ${ }^{12,13}$ Samples were epitaxial with (100) orientation and interfaces were atomically sharp. The data shown here correspond to a sample with $8 \mathrm{~nm}$ thick (7 unit cells) YBCO spacer. All our samples exhibit similar MR behavior up to YBCO thicknesses of $21 \mathrm{~nm}$ (Refs. 14 and 15) and were found to display the same angular dependence discussed here.

Both top and bottom LCMO layers have in-plane easy axes along the [110] direction, as referenced to STO. This was shown by ferromagnetic resonance and by a set of dc magnetization hysteresis loops recorded with fields applied along various in-plane directions in both experiments. ${ }^{15} \mathrm{MR}$ was measured using a four-probe van der Pauw style geom- 

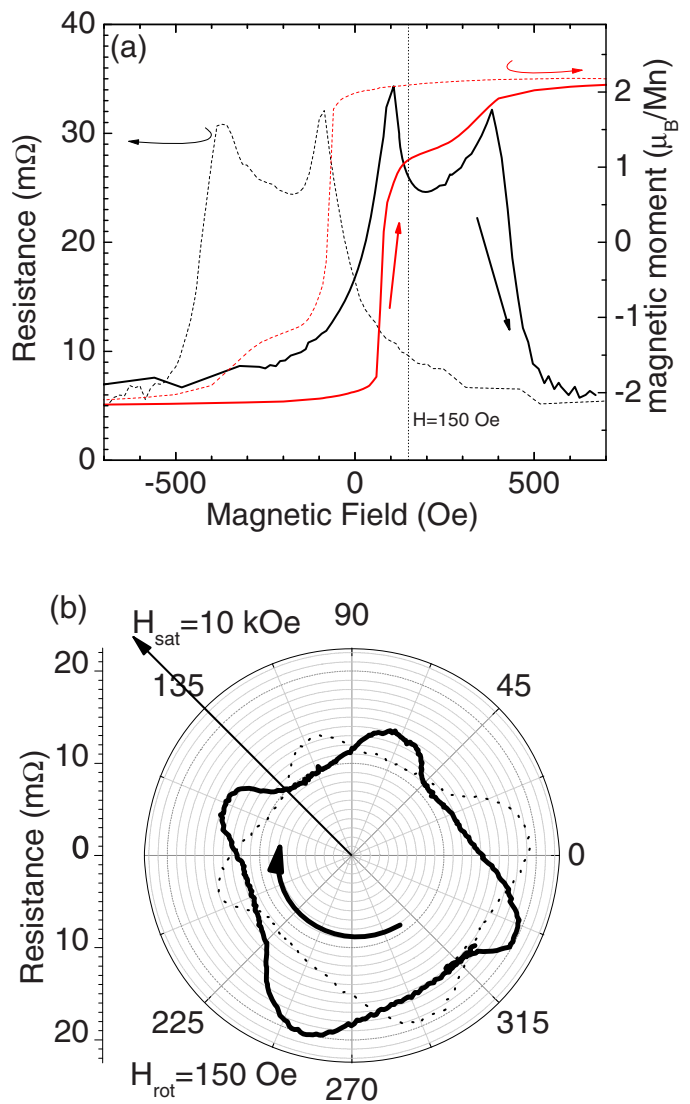

FIG. 1. (Color online) (a) MR and magnetic hysteresis loops for magnetic field applied along [110]. Dotted line indicates $\mathrm{H}_{\mathrm{app}}=150$ Oe. (b) Resistance vs angle $(\theta)$ in $\mathrm{H}_{\text {app }}=150$ Oe after saturating the sample in $10 \mathrm{kOe}$ at $\theta=135^{\circ}$. The thick line shows rotation of $\mathrm{H}_{\text {app }}$ from high-angle to low-angle, while the dotted line is the reverse. The long arrow indicates the direction of the $\mathrm{H}_{\mathrm{sat}}=10 \mathrm{kOe}$ saturating field. Temperature of this measurement was $23 \mathrm{~K}$.

etry with dc current injected in plane. We denote the angle between the magnetic field and the [100] substrate edges (the direction of one of the magnetic hard axes and the dc current, too) by $\theta$.

The typical MR and magnetization hysteresis loops are shown in Fig. 1(a). The effect of magnetic alignment on superconductivity can be closely tracked by the resultant positive MR signals in the AP state. Applying magnetic field along the easy axis enables a well-defined AP state within a broad magnetic field range. The different coercivities of top and bottom layers allow controlling the relative magnetic alignment of the $\mathrm{F}$ layers over a large magnetic field range. ${ }^{14-16}$

Because the (biaxial) magnetic anisotropy modulates the MR in an experiment rotating the magnetic field in the plane of the film, we performed rotation experiments using an applied field $\left(\mathrm{H}_{\mathrm{app}}\right)$ of variable direction. Figure 1(b) shows MR as a function of the in-plane field direction by rotating an applied field with constant magnitude of 150 Oe. This $\mathrm{H}_{\text {app }}$ is larger than the anisotropy field of the bottom layer and smaller than that of the top layer. We describe the magnetization switching with reference to the polar plot of the Stoner-Wohlfarth critical switching curve (CSC) or magnetic astroid. In films with biaxial anisotropy, the CSC is an astroid of eight cusps. ${ }^{17}$ Consider when $\mathrm{H}_{\text {app }}$ of magnitude less than the anisotropy field of the film is applied along its easy axis and then rotated. Then, the magnetization of the film lags progressively further behind the $\mathrm{H}_{\text {app }}$ direction, until the tip of the $\mathrm{H}_{\text {app }}$ vector intersects a point on the CSC at the hard axis. Then, the angle of magnetization with $\mathrm{H}_{\text {app }}$ undergoes a large jump and precedes $\mathrm{H}_{\mathrm{app}}$, until the astroid is intersected again at the next easy axis. For rotations in opposite sense, magnetization jumps at angles symmetric to the nodes of the asteroid, resulting in hysteretic behavior. Since the chosen $\mathrm{H}_{\mathrm{app}}=150$ Oe is between the coercive fields $\mathrm{H}_{\mathrm{c}}$ of bottom $(\mathrm{Hc}=80 \mathrm{Oe})$ and top $\left(\mathrm{H}_{\mathrm{c}}=350 \mathrm{Oe}\right)$ of the two layers, the magnetization of the bottom LCMO can rotate freely, following $\mathrm{H}_{\text {app }}$ while the magnetization of the top LCMO lags behind or precedes it. In Fig. 1(b) we plotted resistance versus angle in $\mathrm{H}_{\text {app }}=150$ Oe after saturating in $\mathrm{H}_{\mathrm{sat}}=10 \mathrm{kOe}$ along $\theta_{\mathrm{sat}}=135^{\circ}$. The direction of the saturating field $\theta_{\text {sat }}$ determines an overall unidirectional (exchange bias-like) anisotropy with a minimum MR at $\theta_{\text {sat }}$ because in this direction the two magnetizations are parallel. Data were taken at $23 \mathrm{~K}$, whereas the zero resistance critical temperature was $\mathrm{T}_{\mathrm{C}}=17 \mathrm{~K}$. The thick line in Fig. 1(b) shows the rotation from high angle to low angle; the thin line shows the return. The peaks coincide with the magnetization jumps when a hard axis is passed by, and exhibit hysteretic (rotational) behavior, i.e., angular positions of the local MR maxima are placed symmetrically on either side of the hard axes for the two senses of rotations. Increasing the field of rotation $\left(\mathrm{H}_{\text {app }}\right)$ brings MR maxima increasingly closer to the hard axes directions (not shown).

MR rotation curves show that $\mathrm{F} / \mathrm{S} / \mathrm{F}$ heterostructures can be used as memory devices, based on the biaxial symmetry that can be described as follows. Operation is determined by the misaligned magnetization as in ordinary F/N/F memory elements with normal metal $(\mathrm{N})$ spacers. Note that in our inverse spin switches (and in F/N/F structures) the high resistance state occurs in the AP configuration contrary to ordinary superconducting spin switches where the AP alignment favors superconductivity and thus a low resistance state. Since interface is atomically sharp, the observed MR is free of the effect of interface roughness observed previously in FNF structures. ${ }^{18,19}$ Therefore the biaxial anisotropy yields stable magnetization states in four perpendicular directions (the easy axes). Moreover, the very large resistance change between P and AP states (50\%), stable over the whole field sweep, enables the use of this memory concept in practical devices.

A demonstration of this memory concept is depicted in Fig. 2. Figure 2(a) shows the angle dependent MR as the $\mathrm{H}_{\text {app }}=150$ Oe writing field is rotated in the film plane after saturating at 135 degrees. This curve is highly reproducible over various rotations, as long as a larger saturating field (over 500 Oe) is not applied in a different direction. The " 0 " or " 1 " bit state, designated by choosing a field angle and corresponding MR, is erased by rotating the writing field. These operations in time are indicated by the vertical lines of Fig. 2(b). As soon as the writing direction is set, the writing field can be removed $\left(\mathrm{H}_{\text {app }}=0\right)$. We chose neighboring directions of local maximum and minimum of the MR curve, as indicated by the large black circle and red square in Fig. 2(a). The read operation consists of fast resistance measurements using a low excitation current of $\mathrm{I}=100 \mu \mathrm{A}$, as shown in Fig. 2(c). Two stable resistance values are obtained that correspond to the two write field angles, indicated by black circles and red squares according to the symbols of panel (a). 

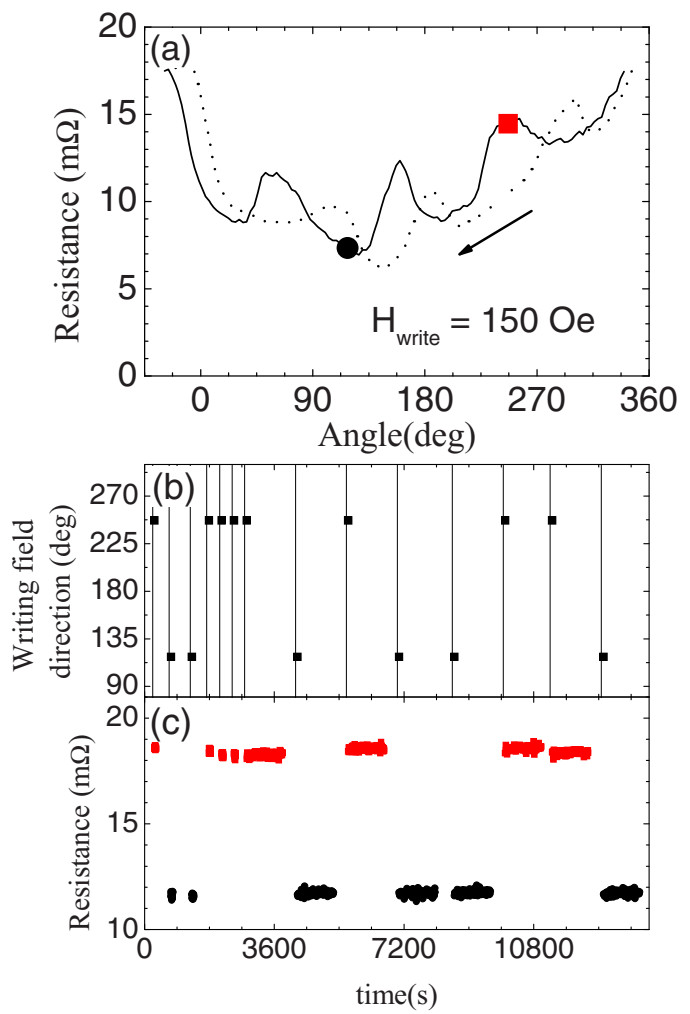

FIG. 2. (Color online) (a) MR as a function of in-plane applied field, $\mathrm{H}_{\mathrm{app}}=150 \mathrm{Oe}$, rotated clockwise (thick line), and counterclockwise (dotted line). The writing positions of the " 0 " and " 1 " states are indicated by the black circle and red square. (b) Writing process. Over time, the in-plane field of $\mathrm{H}=150$ Oe was fully rotated (vertical lines) and then set at the writing angles (black dots). (c) The sample resistance as a function of time, after the write operation and removal of the applied field $\left(\mathrm{H}_{\mathrm{app}}=0\right)$. Temperature of this measurement was $23 \mathrm{~K}$.

These two levels are independent of the order of the write field operations (0's or 1's). They are also stable both over time (days) and over various write operations, with resistance and corresponding voltage level ratios of over $50 \%$. Therefore, this is a good memory element or device concept, where the high and low resistance states are stable at zero field (and thus "memorized") and are read by a simple and fast resistance measurement.

In summary, we implemented an inverse superconducting spin-valve using the high- $\mathrm{T}_{\mathrm{C}}$ superconductor $\mathrm{YBa}_{2} \mathrm{Cu}_{3} \mathrm{O}_{7}$ and highly spin-polarized ferromagnetic $\mathrm{La}_{0.7} \mathrm{Ca}_{0.3} \mathrm{MnO}_{3}$ with a magnetoresistive response controlled by the biaxial in-plane anisotropy. This provides an interesting concept for superconductor based memory elements exploiting a number of peculiarities which differentiates it from GMR based memories. Besides the advantages of the superconductor detection element (fast response and low dissipation) it has the possibility of rather small (100-150 Oe) writing fields applied at different directions and the fast resistance read-out without any applied field. This result represents a significant advance toward the development of superconductor based spintronic device architectures.

We thank A. Goldman for fruitful discussions within the framework of the joint U.S.-Spain NSF Materials World Network Grant No. 709584. Work was supported by the U.S. Department of Energy, Basic Energy Science under Contract Nos. DE-AC02-06CH11357 and DE-AC02NA25396, by Spanish MICINN under Contracts "Ramon y Cajal," Grant Nos. MAT2008-06517 and CONSOLIDER INGENIO 2010 CSD2009-00013 (IMAGINE), by CAM under PHAMA Grant No. S2009/Mat-1756, and by OTKA Grant Nos. K68807 and PF63954 and the "Bolyai" program of the Hungarian Academy of Sciences.

${ }^{1}$ S. Oh, D. Youm, and M. R. Beasley, Appl. Phys. Lett. 71, 2376 (1997). ${ }^{2}$ S. A. Wolf, D. D. Awschalom, R. A. Buhrman, J. M. Daughton, S. von Molnar, M. L. Roukes, A. Y. Chtchelkanova, and D. M. Treger, Science 294, 1488 (2001).

${ }^{3}$ R. Held, J. Xu, A. Schmehl, C. W. Schneider, J. Mannhart, and M. R., Beasley Appl. Phys. Lett. 89, 163509 (2006).

${ }^{4}$ N. M. Nemes, J. E. Fischer, G. Baumgartner, L. Forro, T. Feher, G. Oszlanyi, F. Simon, and A. Janossy, Phys. Rev. B 61, 7118 (2000).

${ }^{5}$ J. Y. Gu, C.-Y. You, J. S. Jiang, J. Pearson, Ya. B. Bazaliy, and S. D. Bader, Phys. Rev. Lett. 89, 267001 (2002).

${ }^{6}$ A. Potenza and C. H. Marrows, Phys. Rev. B 71, 180503(R) (2005).

${ }^{7}$ R. Steiner and P. Ziemann, Phys. Rev. B 74, 094504 (2006).

${ }^{8}$ I. C. Moraru, W. P. Pratt, Jr., and N. O. Birge, Phys. Rev. Lett. 96, 037004 (2006).

${ }^{9}$ A. Y. Rusanov, S. Habraken, and J. Aarts, Phys. Rev. B 73, 060505 (2006).

${ }^{10}$ A. Singh, C. Sürgers, and H. v. Löhneysen, Phys. Rev. B 75, 024513 (2007).

${ }^{11}$ G.-X. Miao, A. V. Ramos, and J. S. Moodera, Phys. Rev. Lett. 101, 137001 (2008).

${ }^{12}$ T. Endo, A. Hoffmann, J. Santamaria, and I. K. Schuller, Phys. Rev. B $\mathbf{5 4}$ R3750 (1996).

${ }^{13}$ V. Peña, Z. Sefrioui, D. Arias, C. Leon, J. Santamaria, J. L. Martinez, S. G. E. te Velthuis, and A. Hoffmann, Phys. Rev. Lett. 94, 057002 (2005).

${ }^{14}$ N. M. Nemes, M. Garcia-Hernandez, S. G. E. te Velthuis, A. Hoffmann, C. Visani, J. Garcia-Barriocanal, V. Peña, D. Arias, Z. Sefrioui, C. Leon, and J. Santamaria, Phys. Rev. B 78, 094515 (2008).

${ }^{15}$ C. Visani, N. M. Nemes, M. Rocci, Z. Sefrioui, C. Leon, S. G. E. te Velthuis, A. Hoffmann, M. R. Fitzsimmons, F. Simon, T. Feher, M. Garcia-Hernandez, and J. Santamaria, Phys. Rev. B 81, 094512 (2010).

${ }^{16}$ Z. Sefrioui, D. Arias, V. Peña, J. E. Villegas, M. Varela, P. Prieto, C. Leon, J. L. Martinez, and J. Santamaria, Phys. Rev. B 67, 214511 (2003).

${ }^{17}$ M. Vogel and T. Mewes, Stoner-Wohlfarth astroid applet, http:// www.bama.ua.edu/ tmewes/Java/Astroid/StonerAstroid.shtml, August 28, 2009.

${ }^{18}$ M. C. Cyrille, S. Kim, M. E. Gomez, J. Santamaria, K. M. Krishnan, and I. K. Schuller, Phys. Rev. B 62, 3361 (2000).

${ }^{19}$ J. Santamaria, M. E. Gomez, J. L. Vicent, K. M. Krishnan, and I. K. Schuller, Phys. Rev. Lett. 89, 190601 (2002). 